Bull. Austral. Math. Soc.

Vol. 40 (1989) [157-160]

\title{
CHEN'S PROBLEM ON MIXED FOLIATE CR-SUBMANIFOLDS
}

\section{MOHAMmed Ali Bashir}

\begin{abstract}
We prove that the simply connected compact mixed foliate $C R$-submanifold in a hyperbolic complex space form is either a complex submanifold or a totally real submanifold. This is the problem posed by Chen.
\end{abstract}

\section{Preliminaries}

Let $\bar{M}$ be an $m$-dimensional hyperbolic complex space form, that is a Kaeller manifold of constant holomorphic sectional curvature-4. The curvature tensor $\bar{R}$ of $\bar{M}$ is given by

$$
\begin{aligned}
\bar{R}(X, Y) Z= & -\{g(Y, Z) X-g(X, Z) Y+g(J Y, Z) J X \\
& -g(J X, Z) J Y+2 g(X, J Y) J Z\}
\end{aligned}
$$

where $J$ is the almost complex structure on $\bar{M}$ and $g$ is the Hermitian metric.

A $(2 p+q)$-dimensional submanifold $M$ of $\bar{M}$ is called a $C R$-submanifold if there exists a pair of orthogonal complementary distributions $D$ and $D^{\perp}$ such that $J \cdot D=D$ and $J D^{\perp} \subset \nu$ where $\nu$ is the normal bundle of $M$ and $\operatorname{dim} D=2 p, \operatorname{dim} D^{\perp}=q[1]$. A $C R$-submanifold is said to be proper if neither $D=\{0\}$ nor $D^{\perp}=\{0\}$. We shall denote by $\bar{\nabla} \nabla, \nabla^{\perp}$ the Riemannian connections on $\bar{M}, M$ and the normal bundle respectively. They are related by

$$
\bar{\nabla}_{X} Y=\nabla_{X} Y+h(X, Y), \bar{\nabla}_{X} N=-A_{N} X+\nabla_{X}^{\frac{1}{X}} N, N \in \nu
$$

where $h(X, Y)$ and $A_{N} X$ are the second fundamental forms satisfying

$$
g(h(X, Y), N)=g\left(A_{N} X, Y\right) .
$$

Denote by $R$ and $R^{\perp}$ the curvature tensors associated with $\nabla$ and $\nabla^{\perp}$ respectively. Then the equations of Codazzi and Ricci are given by.

$$
\begin{aligned}
\bar{R}(X, Y, Z, N) & =g\left(\left(\nabla_{X} h(Y, Z)\right)-\left(\nabla_{Y} h\right)(X, Z), N\right) \\
\bar{R}\left(X, Y ; N, N^{\prime}\right) & =R^{\perp}\left(X, Y ; N, N^{\prime}\right)-g\left(\left[A_{N}, A_{N^{\prime}}\right](X), Y\right)
\end{aligned}
$$

Received 16 November 1988

Copyright Clearance Centre, Inc. Serial-fee code: 0004-9729/89 $\$$ A2.00+0.00. 
where $\left(\nabla_{X} h\right)(Y, Z)=\nabla \frac{1}{X} h(Y, Z)-h\left(\nabla_{X} Y, Z\right)-h\left(\nabla_{X} Z, Y\right) X, Y, Z \in *(M)$ and $N, N^{\prime} \in \nu$.

A $C R$-submanifold is said to be mixed foliate if

(i) $D$ is integrable and

(ii) $h(X, Y)=0, X \in D, Y \in D^{\perp}$.

For a mixed foliate $C R$-submanifold the following hold (see [2])

$$
\begin{aligned}
& h(X, J Y)=h(J X, Y) \text { for } X, Y \in D ; \\
& A_{N} X \in D \text { for } X \in D ; \text { and } A_{N} X \in D^{\perp} \text { for } X \in D^{\perp} ; \\
& A_{N} J X=-J A_{N} X \text { for } X \in D ; \\
& A_{J X} Y=A_{J Y} X \text { for } X, Y \in D^{\perp} ; \\
& \nabla_{X} Y \in D^{\perp} \text { for } X, Y \in D^{\perp} ; \nabla_{X} Y \in D \text { for } X \in D^{\perp}, Y \in D ; \\
& \text { and } \nabla_{X}^{\perp} J Y \in J D^{\perp} \text { for } X \in D, Y \in D^{\perp} .
\end{aligned}
$$

The normal bundle splits as $\nu=J D \oplus \mu$ where $\mu$ is a $J$-invariant sub-bundle of $\nu$.

\section{MAIN TheOREM}

We shall prove the following theorem which was proved by Chen [2] for the case $\operatorname{dim} M \leqslant 5$ and was conjectured for the general case.

TheOREM. Let $M$ be a simply connected compact mixed foliate $C R$-submanifold of a hyperbolic complex space form $\bar{M}(-4)$. Then $M$ is either a complex submanifold or a totally real submanifold of $\bar{M}$.

First we prove the following

LEMMA. Let $M$ be a proper nixed foliate $C R$-submanifold of a hyperbolic complex space form $\bar{M}(-4)$. Then for $X, Y \in D, h(X, Y) \in J D^{\perp}$.

Proof: For $X, Y \in D$ and $Z \in D^{\perp}$ it follows from (1.1) that $[\bar{R}(X, Y) Z]^{\perp}=$ $-2 g(X, J Y) J Z$. Using this and $X, Y \in D$ in the Codazzi equation (1.3) we get

$$
-2 g(X, J Y) J Z=h\left(X, \nabla_{Y} Z\right)-h\left(Y, \nabla_{X} Z\right) .
$$

Taking the inner product with $J W \in J D^{\perp}$ and replacing $X$ by $J X$ we have, using (1.2), (1.6) and (1.7), that

$$
\begin{aligned}
-2 g(J X, J Y) g(J Z, J W) & =g\left(h\left(J X, \nabla_{Y} Z\right), J W\right)-g\left(h\left(Y, \nabla_{J X} Z\right), J W\right) \\
& =g\left(A_{J W} J X, \bar{\nabla}_{Y} Z\right)-g\left(A_{J W} Y, \bar{\nabla}_{J X} Z\right) \\
& =g\left(A_{J W} X, \bar{\nabla}_{Y} J Z\right)-g\left(A_{J W} Y, \bar{\nabla}_{J X} Z\right) \\
& =g\left(A_{J W} X,-A_{J Z} Y\right)-g\left(J_{J W} Y,-\left(A_{J Z} J X\right)\right) \\
& =-g\left(A_{J W} X, A_{J Z} Y\right)-g\left(A_{J W} Y, A_{J Z} X\right) .
\end{aligned}
$$


Thus we get:

$$
2 g(X, Y) g(Z, W)=g\left(A_{J W} X, A_{J Z} Y\right)+g\left(A_{J W} Y, A_{J Z} X\right) .
$$

From (1.9) we get $R^{\perp}(X, Y) J Z \in J D^{\perp}$ for $X, Y \in D$. Thus for $N \in \mu$, the Ricci equation (1.4) and equation (1.1) gives

$$
g\left(\left[A_{J Z}, A_{N}\right](X), Y\right)=0 .
$$

Taking the inner product in (2.1) with $N \in \mu$ and using similar techniques to those used in (2.2) we get

$$
g\left(A_{N} X, A_{J Z} Y\right)+g\left(A_{J Z} X, A_{N} Y\right)=0 .
$$

Combining (2.3) and (2.4) we get

$$
A_{J Z}\left(A_{N} X\right)=0 \text { for } X \in D .
$$

Replacing $X$ by $A_{N} X$ in (2.2) and using the above equation we get

$$
2 g\left(A_{N} X, Y\right) g(Z, W)=0 .
$$

Now suppose that $M$ is a proper $C R$-submanifold. Then the above equation gives $g(h(X, Y), N)=0$, that is $h(X, Y) \in J D^{\perp}$ for $X, Y \in D$.

Proof of THE THEOREM: Let $M$ be a proper mixed foliate $C R$-submanifold of $\bar{M}(-4)$. For any unit vector $Z \in D^{\perp}$ and $X \in D$ we easily get from (1.6) and (2.2) that $A_{J Z}^{2} X=X$. Now take a non-zero vector $Y_{0} \in D$ and consider the set $\left\{J h\left(X, Y_{0}\right): X \in D\right\}$. By the above lemma, this is a subset of $D^{\perp}$. We claim that $D^{\perp}=\left\{J h\left(X, Y_{0}\right): X \in D\right\}$, for if not then there exists a unit vector $Z \in D^{\perp}$ such that $g\left(J h\left(X, Y_{0}\right), Z\right)=0, X \in D$. This gives $g\left(A_{J Z} X, Y_{0}\right)=0$. In particular for $X=A_{J Z} Y_{0}$, we get $g\left(A_{J Z}^{2} Y_{0}, Y_{0}\right)=g\left(Y_{0}, Y_{0}\right)=0$, a contradiction, and hence our claim is established. We can then define the linear map;

$T_{Y_{0}}: D_{p} \stackrel{\text { onto }}{\longrightarrow} D_{p}^{\perp}$ for $p \in M$ given by $T_{Y_{0}} X=J h\left(X, Y_{0}\right)$. Now we prove that $\operatorname{dim} \operatorname{ker} T_{Y_{0}}=2 p-1$. For $X \perp Y_{0}$ we get $0=g\left(Y_{0}, X\right)=g\left(Y_{0}, A_{J Z}^{2} X\right)=$ $g\left(-J h\left(A_{J Z} X, Y_{0}\right), X\right)$, which implies $J h\left(A_{J Z} X, Y_{0}\right)=0$, that is, $A_{J Z} X \in \operatorname{ker} T_{Y_{0}}$. Also with the same argument we would get $A_{J Z} Y_{0} \notin \operatorname{ker} T_{Y_{0}}$. Since $\left.A_{J Z}^{2}\right|_{D}=I$, $\left.A_{J Z}\right|_{D}$ is non-singular, and thus $\operatorname{dim} \operatorname{ker} T_{Y_{0}}=2 p-1$. Then using the fundamental theorem of homomorphism we get $\operatorname{dim} D^{\perp}=1$.

Now let $\xi$ be the unit vector in $D^{\perp}$ which is globally defined on $M$. We shall prove that its dual 1-form $\eta$ is closed. Note that for every vector field $V$ on $M$, we can write

$$
J V=P V+\eta(V) J \xi \text { where } P V \in D .
$$


Then using (1.2) in $\bar{\nabla}_{X} J Y=J \bar{\nabla}_{X} Y$ and equating the $J D^{\perp}$ components we get

$$
(\nabla X \eta)(Y) J \xi=-h(X, P Y)
$$

where we have used the definition of mixed foliate $C R$-submanifold and $h(X, Y) \in J D^{\perp}$ for $X, Y \in D$.

Now using (1.5) we get $d \eta=0$. Since $\eta(\xi)=1$ and $M$ is compact, $\eta$ cannot be exact. Thus $[\eta]$ is a non-trivial cohomology class in the cohomology group $H^{1}(M, \mathbf{R})$. This is contradiction to the simply connectedness of $M$. Hence $M$ cannot be proper and this completes the proof of the theorem

\section{REFERENCES}

[1] A. Bejancu, 'CR-submanifolds of a Kaehler manifold', Proc. Amer. Math. Soc. 69 (1978), 135-142.

[2] B.Y. Chen, ' $C R$-submanifolds of a Kaehler manifold II', J. Differential Geom. 16 (1981), 493-509.

Mathematics Department

College of Science

King Saud University

P.O. Box 2455, Riyadh 11451

Saudi Arabia 